\title{
Guiding Companies to Reduce Technostress: A Mixed-Methods Study Deriving Practice-Oriented Recommendations
}

\author{
Maximilian Valta \\ University of Bamberg \\ maximilian.valta@outlook.de
}

\author{
Katharina Pflügner \\ University of Bamberg \\ katharina.pfluegner@uni- \\ bamberg.de
}

\author{
Christian Maier \\ University of Bamberg \\ christian.maier@uni-bamberg.de
}

\begin{abstract}
Technostress is a major challenge for employees using information technology. Technostress research has revealed the causes, i.e. techno-stressors, and resulting adverse consequences for employees and companies. However, there is a lack of practical insights guiding companies on how to reduce technostress. To offer such practical insights, we follow a mixed-methods approach. The qualitative study bases on eleven expert interviews and reveals seven measures that reduce technostress. We then elaborate on these interview results with a quantitative study of 110 employees. The quantitative results reveal the degree to which the seven measures are useful to reduce specific techno-stressors. Our results show that although there are measures used in practice, none reduces all different techno-stressors. We complement existent theoretical technostress research by offering practice-oriented recommendations on how to reduce technostress. Based on the illustration of which measures are useful for which techno-stressors, practitioners can choose the measures that best fits their needs.
\end{abstract}

\section{Introduction}

A recent study of Microsoft [1] surveys more than 20,000 employees from various small and mediumsized companies and concludes that the usage of information technologies (IT) reduces employees' job satisfaction and job productivity. The reason for this is grounded in employees' experience of technostress. Above all, an overload due to e-mails and text messages is named as specific example of technostress.

Technostress is defined as stress that individuals experience due to their use of IT [2]. It reflects the process of how techno-stressors, defined as events, demands, stimuli, or conditions encountered by individuals as factors that create stress [3, 4], lead to adverse consequences. Adverse consequences among employees include job burnout, low innovativeness, and low job performance $[5,6]$, which lead to health impairment for employees as well as financial losses for companies due to employee absenteeism and lower productivity [7, 8]. Despite these theoretical insights, there is a lack of practical insights for companies on how to reduce techno-stressors and their consequences and thereby the health- and financial-related effects. Therefore, companies search for specific guidance and practice-oriented recommendations to reduce technostress and introduce and organize adequate measures. Thus, we ask:

Which practice-oriented recommendations can be derived for companies to reduce that employees perceive techno-stressors and adverse consequences?

In the following section, we illustrate existing technostress research on techno-stressors and their consequences. Afterwards, we explain the study's methodology and describe the participants of the study. Based on the seven measures that reduce perceptions of techno-stressors introduced in the subsequent section, we derive practice-oriented recommendations for companies.

\section{Techno-stressors and their consequences}

Previous research has focused on a range of different techno-stressors [2]. Techno-overload relates to situations in which the IT forces users to work faster and longer [3, 9]. Techno-invasion represents the invasive effect of the IT in situations in which employees can be reached anytime and anywhere and feel the need to be constantly connected, thus blurring work-related and personal contexts [3]. Techno- 
complexity refers to situations in which the complexity associated with IT leads users to perceive having insufficient IT skills and forces them to spend time and effort on learning and understanding the IT [3]. Techno-insecurity relates to situations in which users feel threatened about losing their jobs, either because of the automation of IT or other people who have a better IT understanding [3]. Techno-uncertainty refers to contexts where continuous IT changes and upgrades unsettle users and create uncertainty so that they must constantly learn and educate themselves about new workflows [3]. Techno-unreliability describes contexts where the IT is not available or does not work as expected [9]. IT-based monitoring represents situations in which employees experience electronic performance monitoring [9]. Cyberbullying describes situations in which employees get offending comments or statements via IT [9]. These eight techno-stressors lead to adverse consequences when the external requirements to reduce the technostressors exceed the employees' internal capabilities [9].

The consequences can be psychological, such as exhaustion and job burnout as well as behavioral, such as low job performance or turnover $[3,5,6]$. These consequences not only affect the individual employees, but also the entire company and its profitability. Therefore, companies need practiceoriented recommendations to reduce technostress among employees and to counteract and prevent the consequences mentioned above. Since there is a recent trend to account for the specific techno-stressors rather than treating them as an aggregate [5], it is reasonable to use specific measures to reduce the different technostressors in practice.

\section{Methodology}

To identify measures that reduce techno-stressors and their consequences, and to develop practiceoriented recommendations for companies on how to introduce and organize these measures, the study follows a mixed-methods approach [10]. We follow the epistemological approach of pragmatism that allows us to combine different research methods, e.g. induction and deduction. Our design strategy can be characterized as sequential, mixed-method and the qualitative and quantitative investigations are equally dominant. So we follow the lead of previous research with similar characteristics [11] and so, we first collected qualitative data in the form of expert interviews, and we, second, distributed a questionnaire to collect data from employees working in three different companies.
We conducted an exploratory internet search for companies that have gained experience with and actively take action to reduce technostress. We thereby identified six companies that implemented internal measures to reduce technostress. We contacted them via e-mail and conducted an initial phone with three companies that agreed to participate in our study and thereby assured that the three companies fulfill the following aspects: First, the companies have been struggling with technostress, e.g. job burnout due to IT implementations. Second, the companies searched for, designed, and implemented measures to reduce technostress. Third, the companies carried out the measures in a structured and evaluative way. For instance, they have introduced a pilot team, a team that tests the measures in practice with only a few employees included, or evaluated the measure's effectiveness by assessing the absence days before and after implementation. This allows us to evaluate the effectiveness as well as dis/advantages of the measures.

Company $A$ is a globally active, listed, diversified industrial company with a focus on steel production and steel processing. Company A employs over 160,000 people worldwide and is headquartered in Germany. Company $B$ is a globally active, listed IT company with numerous subsidiaries. Company B has 380,000 employees and is headquartered in Germany. Company $C$ is an agency for integrated healthcare communication and marketing and cooperates with various globally operating companies. Company $\mathrm{C}$ has about 80 employees and is based in Germany.

We first conducted expert interviews to identify measures taken by the interviewees' companies to reduce technostress. As more and more details emerged in the course of the expert interviews, we second conducted a quantitative study in the form of a questionnaire to elaborate on the qualitative results of the expert interviews and reveal the degree to which these measures are useful to reduce the eight technostressors.

\subsection{Expert interviews}

The semi-structured expert interviews that took place between November 2019 and January 2020 had a duration between 25 and 60 minutes and were conducted by telephone or face-to-face.

To create a common understanding, we defined relevant technostress terms at the beginning and then collected relevant demographic data. We asked the interviewees about measures that are currently used to reduce technostress in the company. Moreover, we also examined the decisive reasons why these measures have been introduced, and how these 
measures have been implemented. We then identified challenges and problems during the implementation phase to assess the dis/advantages of the specific measures and whether a measure successfully reduced technostress.

\subsection{Questionnaire}

We identified seven measures in the expert interviews and designed a questionnaire to further elaborate on these measures. In the questionnaire, we explained each identified measure for reducing technostress and asked the participants to assess the usefulness of this measure to reduce techno-stressors in general and each of the eight techno-stressors in particular (we used a Likert-scale, $1=$ 'not useful at all', $7=$ 'very useful'). A mean value across all answers that is above the mean value of the response scale indicates that the measure is useful. Furthermore, we requested the participants to describe the advantages and disadvantages of the specific measure.

\subsection{Participants}

Table 1 provides an overview of the participants in the expert interviews and questionnaire.

\section{Table 1. Overall participants}

\begin{tabular}{|l|l|l|}
\hline Company & Expert interviews & Questionnaire \\
\hline A & 1 & 48 \\
\hline B & 1 & 37 \\
\hline C & 9 & 25 \\
\hline Total & 11 & 110 \\
\hline
\end{tabular}

Expert interviews. All expert interviewees (Table 2) were involved in the implementation of the measures and have gained experience with technostress, its consequences, and measures to reduce technostress.

Table 2. Expert interviews

\begin{tabular}{|l|l|l|l|l|}
\hline$\#$ & Age & Gender & Position & $\begin{array}{l}\text { Com- } \\
\text { pany }\end{array}$ \\
\hline 1 & 46 & male & press spokesman & A \\
\hline 2 & 42 & male & project manager & B \\
\hline 3 & 41 & male & $\begin{array}{l}\text { executive and project } \\
\text { manager }\end{array}$ & C \\
\hline 4 & 38 & male & quality manager & C \\
\hline 5 & 40 & male & $\begin{array}{l}\text { developer and } \\
\text { technical consultant }\end{array}$ & C \\
\hline 6 & 39 & male & technical consultant & C \\
\hline 7 & 38 & female & project manager & C \\
\hline
\end{tabular}

\begin{tabular}{|l|l|l|l|l|}
\hline 8 & 45 & female & $\begin{array}{l}\text { medical editor and } \\
\text { consultant }\end{array}$ & C \\
\hline 9 & 25 & male & working student & C \\
\hline 10 & 39 & male & concept developer & C \\
\hline 11 & 42 & male & chief executive officer & C \\
\hline
\end{tabular}

Questionnaire participants. We sent the questionnaire to the three companies that were identified for the expert interviews in January 2020 with the request to distribute the questionnaire amongst their employees. 110 employees completed and returned the questionnaire (for demographics see Table 3).

Table 3. Questionnaire participants

\begin{tabular}{|l|l|r|}
\hline \multicolumn{2}{|l|}{ Demographics } \\
\hline Age & $18-25$ & $6.37 \%$ \\
(mean =41.08, & $26-35$ & $21.81 \%$ \\
standard & $36-45$ & $37.27 \%$ \\
deviation =9.67) & $46-55$ & $25.45 \%$ \\
& $>55$ & $9.10 \%$ \\
\hline Gender & Female & $42.73 \%$ \\
\cline { 2 - 3 } & Male & $57.27 \%$ \\
\hline
\end{tabular}

The main fields of occupation are IT (17.27 \%), engineering (13.64 \%), and project management $(10.00 \%)$. All participants estimated their IT usage during work as at least 'rather high', so that IT is present and involved in the daily work.

\section{Measures to reduce technostress}

Based on the qualitative data gained by the expert interviews, we identified seven measures to reduce technostress.

\subsection{Reducing e-mail traffic}

Company A has introduced an e-mail ticket system intending to reduce the distraction of their employees and thus increase productivity. First, Company A intended that the e-mail program delivers e-mails once an hour all e-mails that have been sent throughout the last hour. A test phase with a dedicated pilot team revealed that the tasks of the employees were processed qualitatively better and faster and the employees stated that they were less stressed. In addition to these positive results, the study also revealed a major problem: "Other departments [which had to work with the pilot team] mentioned that this made them work slower because the answers of the pilot team came only once an hour and they often had to waste time with waiting where they could not do any work" (interview partner 1). At this point, the measure 
reduced the productivity of the other departments while at the same time increased the productivity of the pilot team. Company $\mathrm{A}$, therefore, realized that it was not useful to implement this measure only partially.

To resolve the negative effect that employees were not able to respond until the next full hour, a modified system - the e-mail ticket system was implemented, which enables new e-mail conversations to be opened every hour. If a conversation is open, it is possible to answer directly. Hence, every employee is able to write e-mails at any time within this open conversation and there are no restrictions. If an e-mail is delivered and an employee only has a short request, this e-mail is delivered immediately, because the email ticket still has an open status. However, e-mails without an open ticket are only delivered every hour. In this way, issues on one topic can be clarified immediately while employees are not constantly confronted with new topics and less distracted. Additionally, the possibility to close the open ticket manually upon completion was implemented so that the employees do not overrule the e-mail ticket system by using an open conversation for a new topic.

Company $\mathrm{B}$ has a rule to not write e-mails on Sundays and holidays. Likewise, employees are not expected to reply to an e-mail on those days. Also, there is one day per week on which no e-mails need to be answered. Feedback from Company B's employees has shown a positive effect on the employees' daily work by not having to answer e-mails immediately. The feedback has revealed that the employees are more focused on their actual tasks and are less distracted by new tasks incoming via e-mail.

Company $\mathrm{C}$ takes a similar approach. The whole measure is not a strict ban or rule, but rather a corporate culture. Attempts to create a fair corporate culture in the company have been made for a long time. It is endorsed not to read and answer e-mails after work. If there is an important issue, the company's approach is "talk to me and don't write me an e-mail" (interview partners 4, 11), which prevents employees from having to constantly check their emails or being afraid of missing essential ones.

\subsection{Homogenization of IT}

The three companies try to homogenize IT programs and work processes as far as possible. "This means, among other things, trying to provide standardized technologies, both hardware and software, to employees throughout the company to achieve a high level of compatibility. And employees in the commercial departments all receive work laptops. These now all run with the same operating system to avoid heterogeneity. The employees also get business mobile phones and all of them run with Android. This has proven to be a success over the past years. It is done everything to simplify the interfaces and eliminate problems due to incompatibility of software and hardware" (interview partner 2). For the same reason, Microsoft Office and the Microsoft ecosystem are used consistently. The aim is that all employees in the company use consistent software and hardware. In the beginning, there might occur problems and challenges, as many employees are forced to use a new operating system or program which they are not familiar with yet. In this respect, the managers are strict, and the employees have no choice than to use Microsoft Office or Android to establish homogenization. The interviews showed that homogenization of IT lessens complexity and thus reduces technostress for employees. The key is to diminish the variety of programs as much as possible without affecting or complicating communication within the company.

\subsection{Training sessions}

In the three companies, extensive training sessions are offered for employees when new programs, updates or work processes are introduced. These training sessions are partly internal and partly external. Among others, presentations are used to exemplarily show the advantages. Company A also offers regular group meetings, where subject-specific and technical problems are discussed and solved. The goal of such meetings is a quick and uncomplicated solution of occurring problems and there is also time for questions and answers on the latest topics and programs.

\subsection{Introducing contact persons}

The three companies noticed that, despite the training sessions, there are still problems among the employees when new programs are introduced. "For this purpose, a central contact person has been installed, who is available for such issues and problems. This should allow employees to contact someone if they have questions and need help. In the past, some people didn't dare to ask for help, probably because they didn't want to seem stupid, and instead tried to familiarize themselves with the program, which takes a long time and would have been much faster with the help of other people. A central contact person allows everyone to ask for help if there are any problems, without being put in a bad light in front of their teammates, and prevents that other teammates, who would have to help them, are distracted from 
working. Now the employees simply get in touch with the contact person, clarify the issues, and can immediately continue with their work" (interview partner 1). In Company A, the contact person is an employee who has already worked with the newly introduced programs in that company and is willing to support other employees. Problems that cannot be solved by the contact person are usually forwarded e.g. to a central service desk. On the one hand, this measure spreads the workload for this support on many shoulders in the company, but on the other hand, represents an additional effort for the contact persons and can also be a burden for these employees.

\subsection{Mutual support}

The companies aim at cultivating a corporate culture in the way that employees help each other when stress caused by IT arises. For doing so, Company A focuses on the recruiting process and aims at recruiting employees who meet the requirements, e.g. a willingness to help or the ability to work in a team. Company A uses the assessment center to analyze applicants' social skills and whether they fit to the existing team. This should create a more positive and productive work atmosphere in the company in the future. "It has turned out that individual employees in the departments do their job more easily if they simply help each other. It has been shown that it is more efficient and more effective if the employees help each other according to the idea of one helps the other. By that, everyone can benefit from the knowledge of the others. It has proved to be successful in recent years, yielding a huge advantage to the individual employees as well as the individual departments and in fact the entire company. [...] Even if a stupid question comes up, the employee is welcomed with open arms and supported. Meanwhile, the employees clarify issues completely among themselves without supervision" (interview partner 1). The company supports employees during the implementation of new programs and ensures that problems regarding the programs are solved together in a team. Further, the management expects employees to help each other and not only accept but also offer help.

\subsection{Presence days}

Company B noticed technostress among employees due to more and more employees working from home and so the employees working in the office had difficulties to communicate with the teleworking employees. This caused frustration, reduced satisfaction and productivity as well as increased the duration of projects. As a result, Company B has introduced a measure including that on two days a week employees are not allowed to work from home. "The focus here is that employees are supposed to solve problems face-to-face in the company and exchange information about the project within the company. The other three days of the week they can continue working at different locations. This has helped us enormously with our internal communication because the employees can solve problems much easier and faster and don't have to wait long for an answer" (interview partner 2). Due to the implementation of presence days, Company B is now able to simplify the communication between employees, especially on issues involving several employees, thus reducing technostress.

\subsection{Yoga and fitness sessions}

The interviews revealed that relaxing sessions such as yoga and fitness classes are introduced to reduce or prevent technostress and resulting consequences. Such sessions address relaxing and preventing physical problems (e.g. back problems). Company A introduced yoga and fitness sessions gradually. "We start directly in the morning with an approximately 20-minute yoga and fitness session so that the employees start their working day relaxed and rested. The company management realized that there seemed to be a need since many employees attended these sessions and did not want to impede the idea. Meanwhile, three women in Company A run yoga and fitness classes for the employees on an optional basis" (interview partner 1). Furthermore, Company A noticed that the regular yoga and fitness classes have a positive effect on the health of the employees: "We have less stress, back pain, tension since we conduct yoga and fitness classes, and we are really satisfied with our yoga classes. We also had an evaluation of the number of absences from work due to illness, so it is already statistically proven that cases of illness related to back problems, stress, and tension have decreased by 30 percent in the last six months, compared to the six months before, when we did not offer yoga and fitness classes" (interview partner 1).

Company $\mathrm{C}$ also offers yoga sessions, which are held every Monday and Thursday at noon for 15-20 minutes on an optional basis. Additionally, various utensils, sitting and lying facilities are provided in special relaxation rooms. "There are some [employees] who take a nap there when they are tired and have an important appointment in the afternoon" (interview partner 7). 


\section{Evaluation of measures to reduce technostress}

To further evaluate the usefulness of these seven measures to reduce technostress, we conducted quantitative research and distributed an online questionnaire among the employees of the three sampled companies. The participants rated the usefulness of each measure to reduce each specific techno-stressor. The usefulness of the seven measures was rated on a seven-point scale and results are displayed and summarized in Table 4. The colour depicts the degree of usefulness to reduce each specific techno-stressor. For example, the measure contact persons has a usefulness of 6.16 to reduce techno-overload, thus it is the most useful measure to address techno-overload.

Reducing e-mail traffic is very useful to reduce techno-overload and techno-invasion. A homogenization of IT is very useful to reduce technooverload, techno-complexity, and techno-insecurity. Training sessions are very useful to reduce technooverload, techno-complexity, techno-insecurity, and techno-unreliability. Contact persons are useful to reduce techno-overload, techno-complexity, technoinsecurity, techno-uncertainty, and technounreliability. Cooperation and mutual support are useful to reduce techno-overload, techno-complexity, techno-uncertainty, techno-unreliability, and cyberbullying. Presence days and yoga and relaxing sessions are not useful to reduce one of the eight techno-stressors in specific (Table 4).

\section{Table 4. Overview of the results}

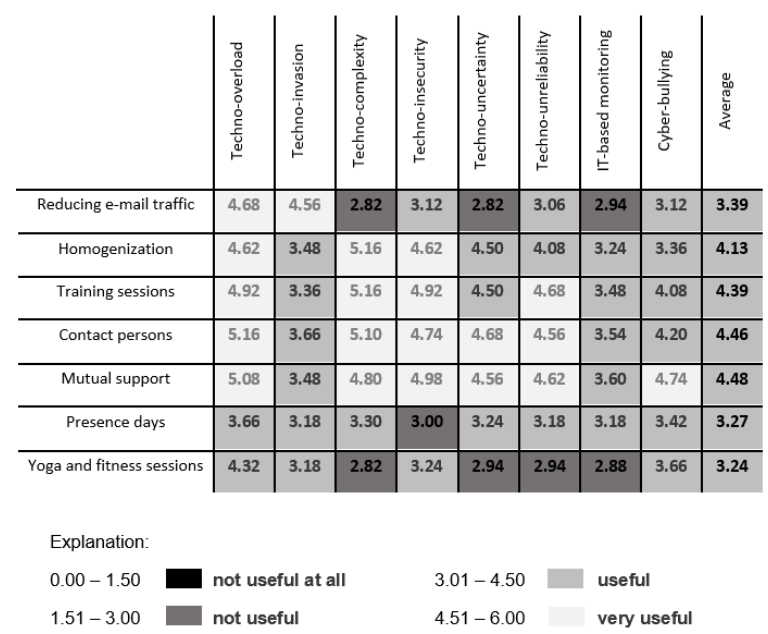

The quantitative results reveal that for each techno-stressor of our study except IT-based monitoring there is at least one measure that is very useful and can be selected to reduce the technostressor.

\section{Practice-oriented recommendations}

In the following, specific recommendations for companies to proactively reduce technostress are presented based on the identified measures and their dis/advantages that came up during the interviews and questionnaire. We recommend a multiple-steps approach, consisting of (a) an as-is analysis, (b) three pillars companies can base on to successfully reduce technostress, i.e. preparing the IT, improving employee skills, and creating a corporate culture, and (c) an evaluation of the effectiveness of the measures to reduce technostress (see Figure 1).

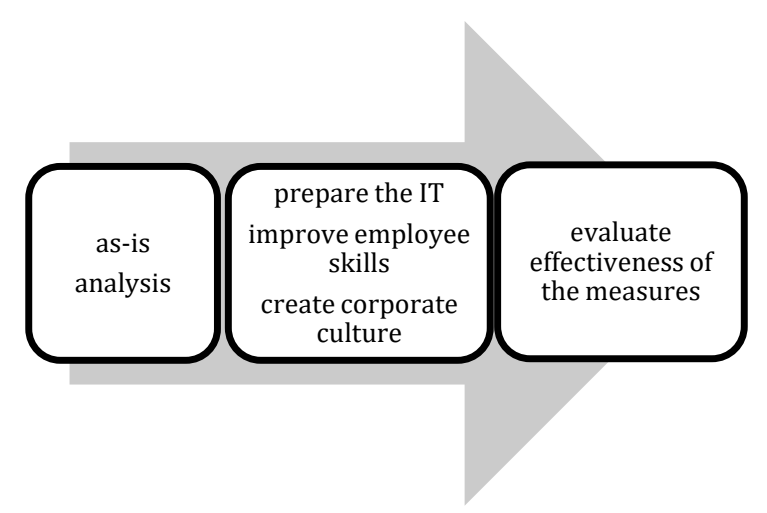

Figure 1. Recommendations to reduce techno-stressors and their consequences

\subsection{As-is analysis}

Our results show that there is no uniform measure to reduce all techno-stressors and for each technostressor to reduce, a different measure is the most useful. Therefore, as a first step, we recommend an as-is analysis to reveal which techno-stressors the employees perceive and should therefore be reduced. Based on the as-is analysis companies should select the measure or a combination of measures that are rated as most useful (see Table 4) to reduce the specific techno-stressor.

\subsection{Prepare the IT}

The first pillar companies can base on to reduce technostress is to prepare the IT, which includes reducing e-mail traffic as well as a homogenization of 
the IT. Preparing the IT should especially be considered when employees perceive technooverload, techno-invasion, techno-complexity or techno-insecurity. IT provides a daily basis for the employees to work, which the company should try to make as simple as possible to reduce technostress.

By reducing e-mail traffic, employees are less distracted from their actual work and can better focus on their current tasks. This is useful to address technooverload. We recommend introducing an e-mail ticket system and clearly explain the advantages of this measure to the employees. Moreover, we suggest explaining that this is not a restriction but an opportunity for the employees and the whole company, and to discuss possible exceptions, e.g. emergency e-mails.

In addition to the e-mail ticket system, shutting down the e-mail servers or establishing the rule "no emails after work and on days off' is very useful for reducing techno-invasion. The aim is to enable employees to disconnect after work. We recommend to establish this rule within the company so that every employee knows about it and respects the fact that no e-mails need to be read or answered after work. To avoid misunderstandings, it is imperative to communicate the information that the e-mail servers will be shut down every day at a certain time. Instead of a long transitional period, we suggest that this rule applies company-wide from a certain day on and that the management sets an example following this rule (see Figure 2).

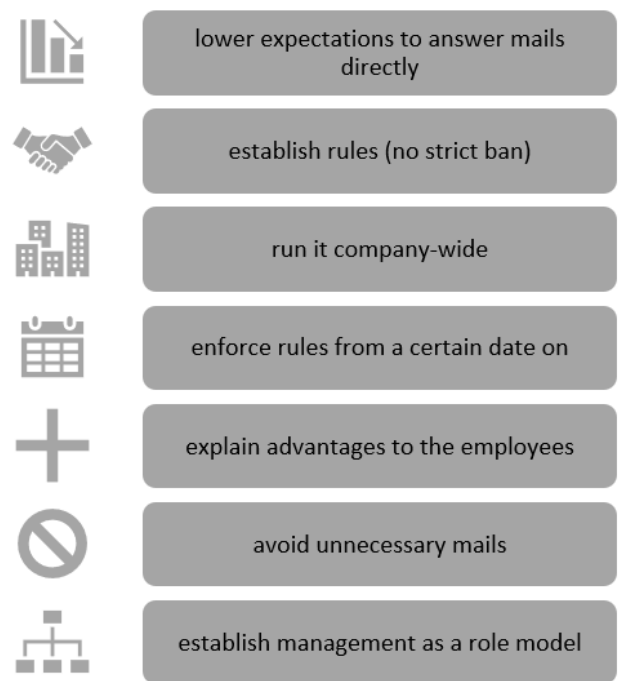

Figure 2. Recommendations for reducing e-mail traffic

Homogenization of IT is very useful to reduce techno-overload, techno-complexity, and techno- insecurity. We recommend companies to agree on a uniform operating system to avoid problems caused by the incompatibility of software and hardware in everyday work and to minimize the number of communication channels. Within the company, at best, only one channel should be used for all internal communication. Here, it is necessary not to commit to one program too fast but to discuss about it with employees from other departments - as they might have different requirements on the tool - and collectively decide on a specific program. Furthermore, we recommend to first test the program with a pilot group to find out about pros and cons and to see whether the program meets the company's requirements before it is rolled out company-wide. By agreeing on a single communication channel in this way, it is possible to avoid employees missing out on information or news because they do not need to keep track of multiple channels. There will certainly be employees who will then have to work with an unfamiliar program or operating system and will be dissatisfied at the beginning, but only if all employees adapt, homogenization can be successfully implemented (see Figure 3).

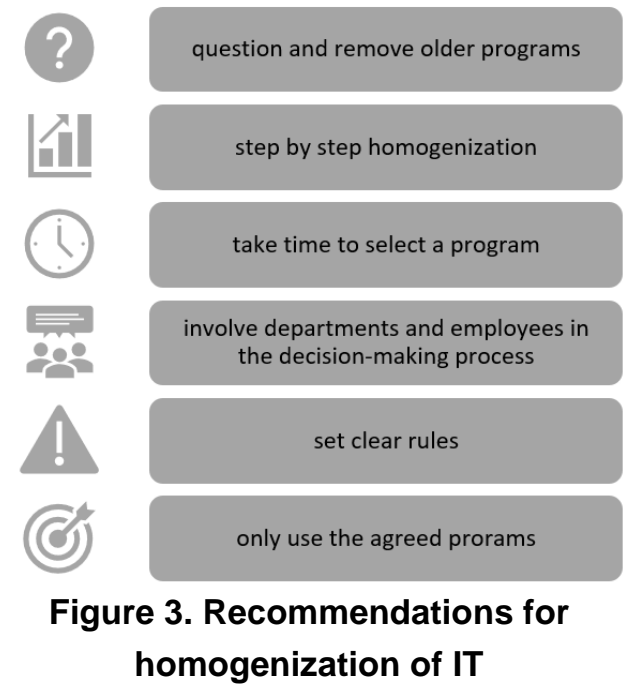

\subsection{Improve employee skills}

The second pillar companies can base on to reduce technostress is to improve their employees' skills, which includes training sessions as well as contact persons. Improving the employee skills should especially be considered when employees perceive techno-overload, techno-complexity, technoinsecurity, techno-uncertainty or techno-unreliability. In this pillar, we recommend to equip the employees with the necessary skills and knowledge to work with 
the IT and to provide on-the-job support for issues that arise at a later point of time.

Analogous to the theoretical idea of literacy facilitation [3,7], which describes mechanisms that try to target the knowledge and skills regarding IT and to increase the company-wide knowledge, we recommend companies to offer training sessions. Concretely, we suggest training sessions along with every implementation of a new program or new work processes to teach employees the advantages and basics of the new program or work processes and make it easier for them to get started. These sessions prevent employees from problems caused by an overload or lack of IT understanding and, in addition, consistently inform employees about the benefits and the way how the program should be used in the company. In this way, employees feel involved and well-informed right from the beginning, which increases their motivation to use the program. Through training sessions, knowledge can be communicated quickly and company-wide, and employees get to know the new program in a group where they can ask questions and do not have to deal with it alone. However, external training courses are expensive and time-consuming. Preferably, there are employees in the company who are familiar with the new program and are willing to give training courses to colleagues. When planning and conducting training sessions it is important to adapt the time and level of instruction to the target audience. Based on the insights we gained throughout the qualitative and quantitative data, we recommend companies to offer different levels of training, e.g. beginners, advanced, experts, to avoid that some employees are overstrained or bored. Likewise, larger training sessions should be divided into several smaller courses up to 15 employees so that employees can learn and practice as much as possible and do not suffer information overload.

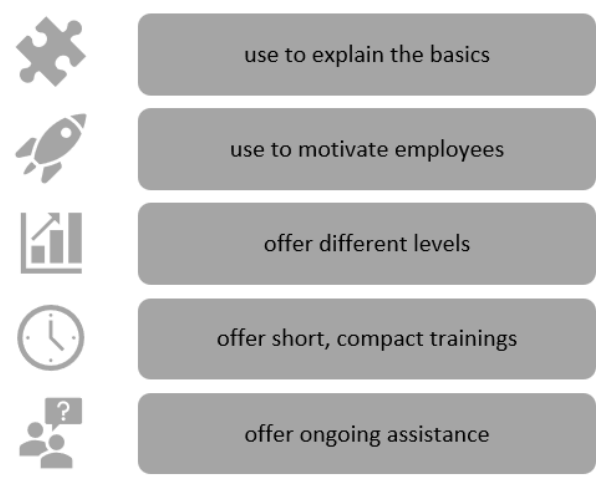

Figure 4. Recommendations for training sessions
However, it is indispensable to make sure that after the implementation of the new program there are still support or contact points for the employees on-the-job, as many problems only arise when the program is in actual use (see Figure 4). The quantitative results show that training sessions are useful to reduce technooverload, techno-complexity, techno-insecurity, and techno-unreliability.

Introducing contact persons reflects the theoretical idea of technical support provision [3, 7], which describes activities aimed at reducing IT-related problems through end-user support. In the introductory training sessions, employees learn the basics and the advantages of the new program. Nevertheless, on-the-job there will appear further problems. We, therefore, recommend companies to provide contact persons in addition to initial training sessions to eliminate individual and later occurring problems and thus relieve their employees. In this way, employees feel supported and know whom to turn to. Contact persons are very useful to reduce techno-overload, techno-complexity, technoinsecurity, techno-uncertainty, and technounreliability because employees thereby get fast and uncomplicated guidance from an expert and do not have to deal with the problems themselves. We suggest keeping the barrier as low as possible so that employees do not shun to seek help from the contact persons, feel supported, and not uncomfortable when they have to search for help. A picture attached to the contact person's profile or a brief introduction of all contact persons in the company helps to keep the barrier low. As far as possible, we recommend installing different contact persons for different areas of expertise and these contact persons should be trained and updated to always be on the state of the art. Contact persons inform themselves about IT-related changes and decide what is important for the whole company and what is not. These information and changes are then filtered and communicated to the employees. In addition to interacting with the employees, contact persons should also interact with each other to identify frequently occurring problems. These problems can then be included in a training session to clarify them in groups and a time-saving way (see Figure 5). 


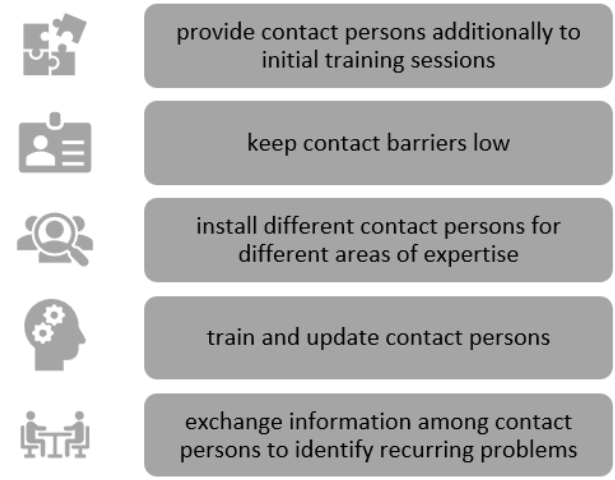

Figure 5. Recommendations for contact persons

\subsection{Create a corporate culture}

The third pillar companies can base on to reduce technostress is to create a corporate culture, which includes mutual support, i.e. to motivate the employees to be proactive to help. Here, a good working atmosphere through mutual support and understanding, for example when e-mails are not answered immediately, is essential.

We recommend establishing a corporate culture of mutual support as it is useful to reduce the majority of techno-stressors, i.e. techno-overload, technocomplexity, techno-uncertainty, techno-unreliability, and cyber-bullying. It is essential to further improve the corporate culture and the self-assessment of the employees. Companies should adapt their corporate culture in such a way that the employees are welcome to ask other employees for advice. There should be an open and helpful atmosphere and management should act as a role model. However, it is also required that the problems can be easily passed on to an expert so that other employees are not unnecessarily distracted from their actual work. Here, attention should be paid to ensuring that employees are able to distinguish between problems they would be better off asking a colleague for help and problems they should rather turn to a central expert (see Figure 6).

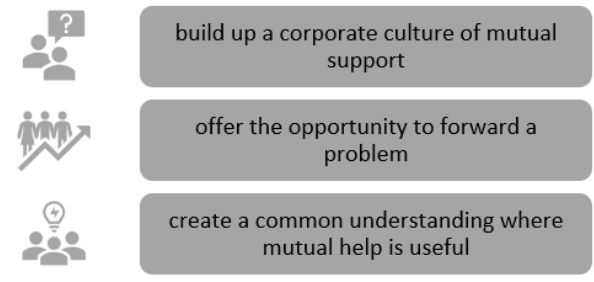

Figure 6. Recommendations for mutual support

\subsection{Measures not directly addressing a techno-stressor}

Although there are two measures, i.e. presence days and yoga and fitness sessions, that are not very useful to directly reduce a specific techno-stressor, we recommend considering these two measures as they may still reduce the adverse consequences of technostressors, e.g. prevent job burnout or increase job satisfaction $[12,13]$.

When introducing presence days, it has to be weighed individually whether presence days can be considered useful or not. If companies find out that e.g. home office causes communication difficulties within teams or departments we recommend introducing days on which all employees have to be on-site in the company. We advise companies to be very strict in this regard and only make a few exceptions, otherwise this advantage will be lost. It is essential to include all employees of the different departments in the decision process on whether and on which days presence days are introduced (see Figure 7). Although our quantitative results do not indicate that the implementation of presence days is very useful to reduce one of the techno-stressors, the expert interviews clearly showed that the implementation of presence days significantly improved internal communication.

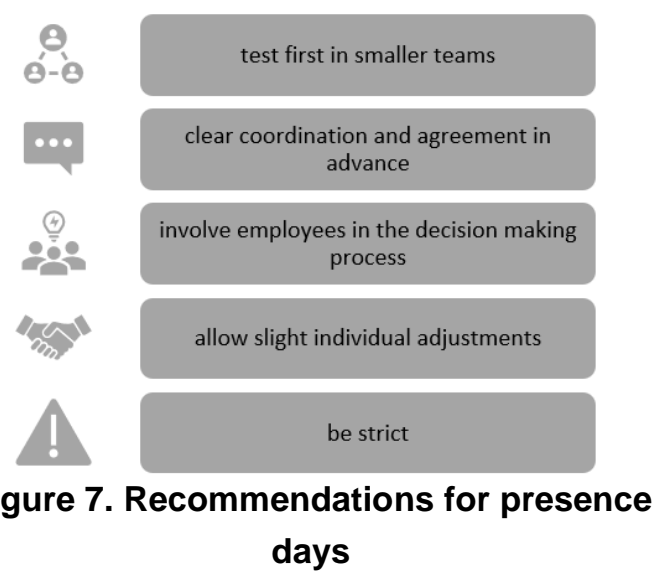

We recommend companies to offer their employees the possibility of yoga and fitness sessions or rest rooms. If possible, the company should offer these classes every day and employees can participate voluntarily. Company B places the yoga classes at the beginning of each working day, but the interviews and questionnaire revealed that it is more effective to offer yoga classes during the lunch break (see Figure 8). Our quantitative results show that yoga and fitness sessions do not reduce any specific techno-stressor but can help to prevent and reduce the consequences of techno- 
stressors, such as muscle tension and back pain as well as job burnout.

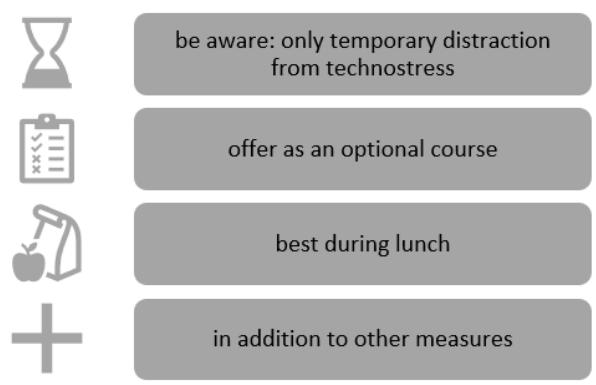

Figure 8. Recommendations for yoga and fitness sessions

\subsection{Evaluate the effectiveness of the measures}

Finally, after implementing these measures, we suggest companies to evaluate the effectiveness of their measures to reduce technostress by regularly conducting surveys assessing employee satisfaction, their perception of technostress, and further status quo analyses. With the help of constant monitoring and adjustment of the measures, technostress can be reduced effectively and sustainably.

\section{Conclusion}

With technostress as ever-increasing challenge, our objective is two-folded. We first aim to identify measures to reduce technostress. Second, we aim to evaluate these measures regarding their usefulness for reducing different techno-stressors. The results of our mixed-methods approach reveal seven measures that reduce technostress and, also, that the choice of the best measure depends on the techno-stressors. The bad news of our research is that there is not one measure that helps to reduce all techno-stressors. However, the good news is that there is at least one useful measure for each techno-stressor. With that in mind, we provide recommendations for organizations and guide them how to introduce and organize the seven identified measures to successfully reduce technostress.

\section{References}

[1] Microsoft (2018): "Digital Culture: Your competitive advantage", https://pulse.microsoft.com/uploads/prod/2018/04/Wor k-Productivity-Digital-Culture-your-competitiveadvantage-Full-research.pdf (Accessed 04.12.2019).

[2] Tarafdar, M., Cooper, C. L., \& Stich, J. F. (2019): "The technostress trifecta-techno eustress, techno distress and design: Theoretical directions and an agenda for research", Information Systems Journal, (29:1), p. 642.

[3] Ragu-Nathan, T. S., Tarafdar, M., Ragu-Nathan, B. S., and Tu, Q. (2008): "The consequences of technostress for end users in organizations: Conceptual development and empirical validation", Information systems research (19:4), p. 417-433.

[4] Pflügner, K., Maier, C., Mattke, J., and Weitzel, T. (2020): "Personaliy Profiles that Put Users at Risk of Perceiving Technostress: A Qualitative Comparative Analysis with the Big Five Personality Traits", Business \& Information Systems Engineering (BISE).

[5] Maier, C., Laumer, S., Wirth, J., and Weitzel, T. (2019):

"Technostress and the hierarchical levels of personality: a two-wave study with multiple data samples", European Journal of Information Systems, (28:5), p. 496-522.

[6] Srivastava, S. C., Chandra, S., and Shirish, A. (2015): "Technostress creators and job outcomes: theorising the moderating influence of personality traits", Information Systems Journal, (25:4), p. 355401.

[7] Tarafdar, M., D’Arcy, J., Turel, O., and Gupta, A. (2015): "The Dark Side of Information Technology," MIT Sloan Management Review (56:2), p. 61-70.

[8] Tarafdar, M., Pullins, E. B., and Ragu-Nathan, T. S. (2015): "Technostress: negative effect on performance and possible mitigations", Information Systems Journal, (25:2), p. 103-132.

[9] Fischer, T., Pehböck, A., and Riedl, R. (2019): "Is the technostress creators inventory still an up-to-date measurement instrument? Results of a large-scale interview study.", Proceedings of the $14^{\text {th }}$ International Conference on Wirtschaftsinformatik.

[10] Venkatesh, V., Brown, S. A., and Bala, H. (2013): "Bridging the qualitative-quantitative divide: Guidelines for conducting mixed methods research in information systems", MIS quarterly, p. 21-54.

[11] Maier, C., Laumer, S., Thatcher, J.B., Sun, H., Weinert, C., and Weitzel, T. (2020): "Social networking site use resumption: A model of return migration", forthcoming in: Journal of the Association for Information Systems (JAIS).

[12] Hülsheger, U. R., Alberts, H. J., Feinholdt, A., and Lang, J. W. (2013): "Benefits of mindfulness at work: the role of mindfulness in emotion regulation, emotional exhaustion, and job satisfaction", Journal of applied psychology, (98:2), p. 310-325.

[13] Pflügner, K., Maier, C., and Weitzel, T. (2020): „The direct and indirect influence of mindfulness on technostressors and job burnout: A quantitative study of white-collar workers", Computers in Human Behavior, 106566. 